\title{
Clinical outcomes and prognostic factors of mortality in liver cirrhosis patients on continuous renal replacement therapy in two tertiary hospitals in Korea
}

\author{
You Hyun Jeon ${ }^{1}$, II Young Kim ${ }^{2,3}$, Gum Sook Jang ${ }^{1}$, Sang Heon Song ${ }^{1,4}$, Eun Young Seong ${ }^{1,4}$, Dong Won Lee ${ }^{2,3}$, \\ Soo Bong Lee $\mathrm{L}^{2,3}$, Hyo Jin Kim ${ }^{1,4}$ \\ ${ }^{1}$ Department of Internal Medicine, Pusan National University Hospital, Busan, Republic of Korea \\ ${ }^{2}$ Department of Internal Medicine, Pusan National University Yangsan Hospital, Yangsan, Republic of Korea \\ ${ }^{3}$ Research Institute for Convergence of Biomedical Science and Technology, Pusan National University Yangsan Hospital, Yangsan, Republic of \\ Korea \\ ${ }^{4}$ Biomedical Research Institute, Pusan National University Hospital, Busan, Republic of Korea
}

\begin{abstract}
Background: Data on liver cirrhosis (LC) patients undergoing continuous renal replacement therapy (CRRT) are lacking despite of the dismal prognosis. We therefore evaluated clinical characteristics and predictive factors related to mortality in LC patients undergoing CRRT.

Methods: We performed a retrospective observational study at two tertiary hospitals in Korea. A total of 229 LC patients who underwent CRRT were analyzed. Patients were classified into survivor and non-survivor groups. We used multivariable Cox regression analyses to identify predictive factors of in-hospital mortality.

Results: During a median follow-up of 5 days (interquartile range, 1-19 days), in-hospital mortality rate was $66.4 \%$. In multivariable analysis, the Acute Physiology and Chronic Health Evaluation II (APACHE II) score (hazard ratio [HR], 1.03; 95\% confidence interval [Cl], 1.01-1.06; $p=0.02$ ), Model for End-Stage Liver Disease (MELD) score (HR, 1.08; 95\% Cl, 1.04-1.11; $p<0.001$ ), and delivered CRRT dose (HR, 0.95; 95\% Cl, 0.92-0.98; $p=0.002)$ were significant risk factors for in-hospital mortality. Patients with a CRRT delivered dose $<25 \mathrm{~mL} / \mathrm{kg} / \mathrm{hr}$ had a higher mortality rate than those with a delivered dose $>35 \mathrm{~mL} / \mathrm{kg} / \mathrm{hr}$ (HR, 3.13; 95\% Cl, 1.62-6.05; $p=0.001$ ). Subgroup analysis revealed that a CRRT delivered dose $<25 \mathrm{~mL} / \mathrm{kg} / \mathrm{hr}$ was a significant risk factor for in-hospital mortality among LC patients with a MELD score $\geq 30$.

Conclusion: High APACHE II score, high MELD score, and low delivered CRRT dose were significant risk factors for in-hospital mortality. CRRT delivered dose impacted mortality significantly, especially in patients with a MELD score $\geq 30$.
\end{abstract}

Keywords: Acute kidney injury, Continuous renal replacement therapy, Liver cirrhosis, Mortality, Prognosis

Received: February 6, 2021, Revised: May 13, 2021, Accepted: May 29, 2021

Editor: Hyeong Cheon Park, Yonsei University, Seoul, Republic of Korea

Correspondence: Hyo Jin Kim

Department of Internal Medicine, Pusan National University Hospital, 179 Gudeok-ro, Seo-gu, Busan 49241, Republic of Korea.

E-mail: kimhj923@gmail.com

ORCID: https://orcid.org/0000-0001-9289-9073

You Hyun Jeon and II Young Kim contributed equally to this manuscript as co-first authors.

Copyright (C) 2021 by The Korean Society of Nephrology

(a) This is an Open Access article distributed under the terms of the Creative Commons Attribution Non-Commercial and No Derivatives License (http:// creativecommons.org/licenses/by-nc-nd/4.0/) which permits unrestricted non-commercial use, distribution of the material without any modifications, and reproduction in any medium, provided the original works properly cited. 


\section{Introduction}

Acute kidney injury (AKI) is a well-known complication of liver cirrhosis (LC) that occurs in approximately $50 \%$ of patients and is strongly associated with increased mortality [1]. Continuous renal replacement therapy (CRRT) has become an important kidney replacement therapy (KRT) modality in the intensive care unit (ICU) setting worldwide [2]. Cirrhotic patients with acute illness frequently experience intravascular volume depletion and hepatic encephalopathy resulting from splanchnic arterial vasodilatation and impaired detoxification of ammonia [3,4]. Under these circumstances, CRRT could be the best KRT option for LC patients because it can maintain hemodynamic stability, prevent elevated intracranial pressure, and reduce serum ammonia levels [5].

Patient prognosis has improved as the proportion of patients undergoing CRRT in the ICU has increased [2]. A recent national epidemiological study in Korea showed that use of CRRT as an AKI treatment has increased over time (2005 to 2007, 4,667 patients [62\%]; 2014 to 2016, 13,414 patients [80\%]), whereas the in-hospital mortality rate has simultaneously decreased (2005 to 2007, 63.4\%; 2014 to 2016, $53.7 \%$ ) [6]. Similarly, in a previous report from our hospital, the short-term mortality rate of patients for whom CRRT was initiated in the ICU was 57.3\% [7]. Despite these changes, the mortality rate of cirrhotic patients who require dialysis due to liver failure but who are noncandidates for liver transplantation remains extremely high at above $80 \%[8,9]$.

Traditionally, KRT has been considered to be a bridging therapy in patients with hepatorenal syndrome (HRS) who are possible candidates for liver transplantation [10]. Providing KRT to acute tubular necrosis (ATN) patients who suffer multiple organ failure has been considered inappropriate. Due to the lack of outcome data for noncandidates for liver transplantation with AKI undergoing KRT, it is difficult to determine whether KRT should be offered to these non-listed patients [11]. However, the widespread use of CRRT has resulted in cirrhotic patients undergoing CRRT in the form of acute KRT. Efforts have recently been made to explore the clinical outcomes of LC patients with AKI. Since the International Club of Ascites Criteria for AKI (ICA-AKI) staging system was adopted, several studies have reported that mortality rate increases with AKI staging [12,13]. Although some studies have analyzed mortality rates according to AKI type, the results have been inconsistent [14,15]. Furthermore, use of different inclusion criteria for AKI staging complicates comparison of study findings. Literature on LC patients with AKI is difficult to generalize because of the variability in regional practice patterns and available medical treatment options such as terlipressin [3]. Clinical outcomes for this highrisk population in Korea are lacking. Therefore, our goal in this study was to evaluate the clinical characteristics and risk factors for in-hospital mortality in non-listed LC patients receiving CRRT.

\section{Methods}

\section{Study subjects}

This was a retrospective study based on clinical data obtained from LC patients admitted to Pusan National University Hospital from January 2013 to December 2018 and to Pusan National University Yangsan Hospital from January 2017 to February 2020. Initially, we investigated all LC patients who started CRRT for the first time during their hospitalization. Exclusion criteria were as follows: (1) subjects under the age of 18 years; (2) subjects who underwent a liver transplantation; and (3) subjects who had undergone maintenance hemodialysis. Patients were classified into survivor and non-survivor groups.

We received appropriate approval from the Institutional Review Board (IRB) of Pusan National University Hospital (No. 1909-011-083) and the IRB of Pusan National University Yangsan Hospital (No. 05-2020-097). The IRBs waived the requirement for informed consent because of the retrospective design of the study.

\section{Data collection and definitions}

We collected demographic data and information on comorbidities, including diabetes, hypertension, and chronic kidney disease (CKD), from electronic medical records. We also obtained the last serum creatinine value within 3 months before admission. CKD was defined as a baseline estimated glomerular filtration rate (eGFR) $<60 \mathrm{~mL} / \mathrm{min} / 1.73 \mathrm{~m}^{2}$. Clinical data, laboratory results, and radiologic findings were reviewed to identify complications related to LC and to ascertain causes of AKI. To estimate the severity of acute illness, Sequential Organ Failure Assessment (SOFA) and Acute Physiology and Chronic Health Evaluation II (APACHE II) 
scores were calculated on the day of ICU admission [16,17]. The severity of LC was assessed using the Model for EndStage Liver Disease (MELD) score [18], which was obtained at the time of CRRT initiation. The application of CRRT was based on clinical judgment by a treating physician. Study investigators reviewed medical records to confirm the diagnosis and need for KRT. The delivered dose of CRRT, expressed as $\mathrm{mL} / \mathrm{kg} / \mathrm{hr}$, was calculated by effluent flow.

The diagnosis of HRS was based on the 2015 ICA-AKI and was as follows [19]: (1) diagnosis of cirrhosis and ascites; (2) diagnosis of AKI according to ICA-AKI criteria; (3) no response after two consecutive days of diuretic withdrawal and plasma volume expansion with albumin at $1 \mathrm{~g} / \mathrm{kg}$ body weight; (4) absence of shock; (5) no current or recent use of nephrotoxic drugs; and (6) no macroscopic signs of structural kidney injury. Shock was defined as a systolic blood pressure of less than $80 \mathrm{mmHg}$ or use of vasopressors. Gastrointestinal bleeding complications were also evaluated. Other LC complications were ascites, lactic acidosis, spontaneous bacterial peritonitis, hepatic encephalopathy, hepatocellular carcinoma, and bleeding episodes except gastrointestinal bleeding due to coagulopathy.

\section{Continuous renal replacement therapy prescription}

CRRT was applied to critically ill LC patients with AKI who had acidemia, hyperkalemia, pulmonary edema, hepatic encephalopathy, or needed solute removal according to the judgment of their physicians. CRRT was performed using the Prismaflex set with the AN 69 ST 100 membrane (Baxter International Inc., Deerfield, IL, USA) in continuous venovenous hemodiafiltration mode. The initial prescribed dose of CRRT ranged from 30 to $40 \mathrm{~mL} / \mathrm{kg} / \mathrm{hr}$ in most cases. Additional modifications were made according to the catabolic state or the presence of metabolic acidosis or hyperkalemia. Heparin-free, heparin, or nafamostat mesylate anticoagulants were selected depending on patient bleeding tendency.

\section{Outcomes}

Study subjects were followed up until discharge, transfer to another hospital, or death in the study hospital. Primary outcome was in-hospital, all-cause mortality during the study period.

\section{Statistical analysis}

Continuous variables are presented as means \pm standard deviations or medians (interquartile range). Categorical variables are presented as numbers (percentages). The Kolmogorov-Smirnov test was performed to assess if variables followed a normal distribution. We performed the $t$ test or Mann-Whitney U test to assess the significance of differences in continuous variables between survivors and non-survivors, and the chi-square test to assess the significance of differences in categorical variables between these two groups. We used natural log transformation to normalize urine output (UO) and CRRT duration values. Mortality according to the reason for requiring CRRT and delivered CRRT dose was analyzed using the Kaplan-Meier method with a log-rank test. CRRT delivered dose was analyzed as both a continuous and categorical variable and patients were divided into three groups according to CRRT delivered dose: $<25,25-35$, and $>35 \mathrm{~mL} / \mathrm{kg} / \mathrm{hr}$ groups. We used univariable and multivariable Cox regression analyses to identify risk factors for in-hospital mortality. Multivariable analysis was performed by selecting variables that showed significance in the univariable analysis, excluding the component variables of the APACHE II or MELD scores except for sex and age. Additionally, we performed subgroup analysis according to LC severity based on the MELD score. We also used the maximum Youden's index in the receiver operating characteristic (ROC) curve to assess the optimal cutoff value of the MELD score. Associations are presented as hazard ratios (HRs) with corresponding 95\% confidence intervals (CIs). All collected data were analyzed using IBM SPSS for Windows version 23.0 (IBM Corp., Armonk, NY, USA). The p-values less than 0.05 were considered statistically significant.

\section{Results}

\section{Clinical characteristics of patients}

A total of 229 LC patients who did not undergo liver transplantation but who underwent CRRT at two tertiary hospitals were analyzed. Patients were grouped into survivors ( $\mathrm{n}=$ 77) and non-survivors $(n=152)$. The baseline characteristics of the study patients are shown in Table 1. Mean age was $58.1 \pm 10.5$ years, and 179 patients $(78.2 \%)$ were male. The proportion of patients with CKD was evaluated based on 172 
Table 1. Baseline clinical characteristics of liver cirrhosis patients undergoing CRRT

\begin{tabular}{|c|c|c|c|c|}
\hline Characteristic & Total & Survivor & Non-survivor & p-value \\
\hline No. of patients & 229 & 77 & 152 & \\
\hline Age (yr) & $58.1 \pm 10.5$ & $57.8 \pm 10.2$ & $58.2 \pm 10.6$ & 0.78 \\
\hline Male sex & $179(78.2)$ & $62(80.5)$ & $117(77.0)$ & 0.54 \\
\hline \multicolumn{5}{|l|}{ Comorbidity } \\
\hline Diabetes mellitus & $74(32.3)$ & $21(27.3)$ & $53(34.9)$ & 0.25 \\
\hline Hypertension & $70(30.6)$ & $24(31.2)$ & $46(30.3)$ & 0.89 \\
\hline$C K D^{a}$ & $24(14.0)$ & $6(3.5)$ & $18(10.5)$ & 0.27 \\
\hline \multicolumn{5}{|l|}{ ICU risk factor } \\
\hline Ventilator use & $123(53.7)$ & $36(46.8)$ & $87(57.2)$ & 0.13 \\
\hline Vasopressor use & $158(69.0)$ & $38(49.4)$ & $120(78.9)$ & $<0.001$ \\
\hline $\mathrm{SBP}(\mathrm{mmHg})$ & $107 \pm 22$ & $117 \pm 21$ & $103 \pm 21$ & $<0.001$ \\
\hline SOFA score & $13.0 \pm 4.8$ & $11.2 \pm 4.2$ & $14.0 \pm 4.8$ & $<0.001$ \\
\hline APACHE II score & $26.0 \pm 7.2$ & $23.9 \pm 6.3$ & $27.1 \pm 7.4$ & 0.002 \\
\hline MELD score & $31.9 \pm 5.6$ & $29.4 \pm 5.4$ & $33.2 \pm 5.2$ & $<0.001$ \\
\hline UO for $6 \mathrm{hr}$ before CRRT $(\mathrm{mL})$ & $80(20-183)$ & $125(30-365)$ & $60(20-144)$ & 0.002 \\
\hline CRRT duration (hr) & $35.0(16.0-91.0)$ & $36.0(15.0-101.0)$ & $32.0(16.3-83.5)$ & 0.44 \\
\hline \multicolumn{5}{|l|}{ CRRT prescription } \\
\hline CRRT downtime (hr) & $1.0(0.0-4.5)$ & $1.0(0.0-4.5)$ & $1.0(0.0-4.8)$ & 0.76 \\
\hline Prescribed dose $(\mathrm{mL} / \mathrm{kg} / \mathrm{hr})$ & $38.9 \pm 4.8$ & $39.7 \pm 5.0$ & $38.5 \pm 4.7$ & 0.08 \\
\hline Delivered dose (mL/kg/hr) & $33.4 \pm 6.0$ & $35.1 \pm 5.5$ & $32.4 \pm 6.0$ & 0.001 \\
\hline \multicolumn{5}{|l|}{ Laboratory finding } \\
\hline White blood cell $\left(10^{3} / \mu \mathrm{L}\right)$ & $13.8 \pm 9.8$ & $12.4 \pm 7.0$ & $14.5 \pm 11.0$ & 0.09 \\
\hline Hemoglobin (g/dL) & $9.4 \pm 2.4$ & $9.4 \pm 2.1$ & $9.4 \pm 2.5$ & 0.97 \\
\hline Platelet $\left(10^{3} / \mu \mathrm{L}\right)$ & $86.1 \pm 61.2$ & $93.0 \pm 59.5$ & $82.6 \pm 62.0$ & 0.23 \\
\hline Total bilirubin (mg/dL) & $5.7 \pm 7.0$ & $4.9 \pm 5.2$ & $6.1 \pm 7.8$ & 0.21 \\
\hline Total protein (g/dL) & $5.4 \pm 1.1$ & $5.6 \pm 1.2$ & $5.2 \pm 1.1$ & 0.02 \\
\hline Albumin (g/dL) & $2.8 \pm 1.7$ & $2.8 \pm 0.8$ & $2.8 \pm 2.0$ & 0.79 \\
\hline Creatinine (mg/dL) & $3.6 \pm 6.0$ & $3.6 \pm 2.3$ & $3.5 \pm 7.1$ & 0.99 \\
\hline BUN (mg/dL) & $49.5 \pm 30.3$ & $47.0 \pm 29.7$ & $50.7 \pm 30.6$ & 0.39 \\
\hline Sodium (mmol/L) & $134.0 \pm 8.1$ & $132.1 \pm 6.0$ & $135.0 \pm 8.9$ & 0.004 \\
\hline PT-INR & $2.4 \pm 1.5$ & $2.1 \pm 0.9$ & $2.6 \pm 1.7$ & 0.004 \\
\hline Total $\mathrm{CO}_{2}(\mathrm{mmol} / \mathrm{L})$ & $13.4 \pm 6.6$ & $13.6 \pm 7.1$ & $13.3 \pm 6.3$ & 0.77 \\
\hline $\mathrm{pH}$ & $7.3 \pm 0.1$ & $7.3 \pm 0.1$ & $7.2 \pm 0.1$ & 0.02 \\
\hline \multicolumn{5}{|l|}{ Causes of CRRT } \\
\hline HRS & $18(7.9)$ & $3(3.9)$ & $15(9.9)$ & 0.11 \\
\hline \multicolumn{5}{|l|}{ Acute kidney injury } \\
\hline With shock & $172(75.1)$ & $57(74.0)$ & $115(75.7)$ & 0.79 \\
\hline Without shock & $39(17.0)$ & 17 (22.1) & $22(14.4)$ & 0.15 \\
\hline
\end{tabular}

Data are expressed as number only, mean \pm standard deviation, number (\%), or median (interquartile range).

APACHE II, Acute Physiology and Chronic Health Evaluation II; BUN, blood urea nitrogen; CRRT, continuous renal replacement therapy; HRS, hepatorenal syndrome; ICU, intensive care unit; MELD, Model for End-Stage Liver Disease; PT-INR, prothrombin time-international normalized ratio; SBP, systolic blood pressure; SOFA, Sequential Organ Failure Assessment; UO, urine output.

${ }^{a}$ The proportion of patients with CKD was evaluated based on 172 patients with an available baseline estimated glomerular filtration rate value.

patients with an available baseline eGFR value. Twenty-four patients (14.0\%) had underlying CKD, of which 23 patients had advanced CKD (eGFR category of G3b or higher). We presented baseline, admission, and peak serum creatinine values in Supplementary Table 1 (available online). There were no significant differences in age, sex, or comorbidities 
between survivor and non-survivor groups. The most common cause of admission was infection (25.8\%) followed by other LC complications (24.5\%), gastrointestinal (GI) bleeding (17.0\%), HRS or AKI (9.6\%), cardiovascular events (5.7\%), and other factors such as trauma or transcatheter arterial chemoembolization (17.5\%).

On initiation of CRRT, 123 (53.7\%) and 158 patients (69.0\%) required mechanical ventilation and vasopressors, respectively. Not only was the use of vasopressors significantly higher ( $49.4 \%$ vs. $78.9 \%$, respectively; $\mathrm{p}<0.001$ ) but systolic blood pressure was significantly lower $(117 \pm 21 \mathrm{mmHg}$ vs. $103 \pm 21 \mathrm{mmHg}$, respectively; $\mathrm{p}<0.001)$ in the non-survivor group than the survivor group. The survivor group had lower SOFA and APACHE II scores on ICU admission and a lower MELD score on initiation of CRRT than the non-survivor group (11.2 \pm 4.2 vs. $14.0 \pm 4.8$, respectively, $\mathrm{p}<0.001$; $23.9 \pm 6.3$ vs. $27.1 \pm 7.4$, respectively, $\mathrm{p}=0.002 ; 29.4 \pm 5.4$ vs. $33.2 \pm 5.2$, respectively, $\mathrm{p}<0.001$ ). The survivor group had a higher UO for the 6 hours before initiation of CRRT than the non-survivor group (125 mL [30-365 mL] vs. $60 \mathrm{~mL}$ [20-144 $\mathrm{mL}$ ], respectively; $\mathrm{p}=0.002$ ). The prescribed dose of CRRT was similar between the survivor and non-survivor groups $(39.7 \pm 5.0 \mathrm{~mL} / \mathrm{kg} / \mathrm{hr}$ vs. $38.5 \pm 4.7 \mathrm{~mL} / \mathrm{kg} / \mathrm{hr}$, respectively; $\mathrm{p}$ $=0.08$ ); however, the actual delivered dose was higher in the survivor group than in the non-survivor group (35.1 \pm 5.5 $\mathrm{mL} / \mathrm{kg} / \mathrm{hr}$ vs. $32.4 \pm 6.0 \mathrm{~mL} / \mathrm{kg} / \mathrm{hr}$, respectively; $\mathrm{p}=0.001$ ). Sodium and prothrombin time-international normalized ratio (PT-INR) levels were significantly lower (132.1 $\pm 6.0 \mathrm{mmol} /$ L vs. $135.0 \pm 8.9 \mathrm{mmol} / \mathrm{L}$, respectively, $\mathrm{p}=0.004 ; 2.1 \pm 0.9$ vs. $2.6 \pm 1.7$, respectively, $\mathrm{p}=0.004$ ) in survivors, whereas total protein was significantly higher in survivors compared with non-survivors $(5.6 \pm 1.2 \mathrm{~g} / \mathrm{dL}$ vs. $5.2 \pm 1.1 \mathrm{~g} / \mathrm{dL}$, respectively; $\mathrm{p}=0.020$ ). The leading indication for CRRT was AKI with shock (75.1\%). The proportion of HRS tended to be higher in non-survivors than survivors (9.9\% vs. $3.9 \%$, respectively). However, no statistically significant differences in reason for requiring CRRT were observed between the two groups.

\section{Risk factors for in-hospital mortality}

Causes of death are presented in Table 2. During a median follow-up period of 5 days (interquartile range, 1-19 days), the in-hospital mortality rate was $66.4 \%$. Twenty-one patients (9.2\%) died within 24 hours of being admitted to the ICU. The most common causes of death were LC complica- tions (36.2\%) and infection (22.4\%), except for spontaneous bacterial peritonitis. The Kaplan-Meier curve showed that the cumulative survival rate was not significantly different according to the CRRT causes of HRS, AKI with shock, and AKI without shock (Fig. 1). Cumulative survival rate was also similar between the HRS and AKI groups ( $\mathrm{p}=0.26)$.

Table 3 shows the Cox regression analysis results for in-hospital mortality. In univariable analysis, indicators of acute disease severity such as SOFA score (HR, 1.06; 95\% CI, 1.03-1.10; $\mathrm{p}<0.001$ ), APACHE II score (HR, 1.04; 95\% CI, 1.02-1.06; $\mathrm{p}=0.001$ ), and MELD score (HR, 1.08; 95\% CI, 1.05-1.11; $\mathrm{p}<0.001)$ were significantly correlated with increased in-hospital mortality. Low log UO, log CRRT duration, and CRRT delivered dose were also associated with increased in-hospital mortality (HR, 0.90; 95\% CI, 0.83-0.98; $\mathrm{p}=0.01 / \mathrm{HR}, 0.77 ; 95 \% \mathrm{CI}, 0.68-0.87 ; \mathrm{p}<0.001 /$ and HR, 0.95; 95\% CI, 0.92-0.98; $\mathrm{p}=0.001$, respectively). In multivariable Cox regression analysis, APACHE II score (HR, 1.03; 95\% CI, 1.01-1.06; $\mathrm{p}=0.02$ ), MELD score (HR, 1.08; 95\% CI, 1.04-1.11; $\mathrm{p}<0.001$ ), and delivered CRRT dose (HR, 0.95; 95\% CI, 0.92-0.98; $\mathrm{p}=0.002$ ) were significant risk factors for in-hospital mortality. We further investigated the association with in-hospital mortality by using the CRRT delivered dose as a categorical variable. Kaplan-Meier curves for cumulative survival rates stratified by CRRT delivered dose are presented in Fig. 2. When comparing the three CRRT delivered dose groups, the survival rate was lowest in the group with a CRRT delivered dose $<25 \mathrm{~mL} / \mathrm{kg} / \mathrm{hr}$ (log-rank $\mathrm{p}<0.001$ ). The mortality rate was significantly higher in patients with a CRRT delivered dose $<25 \mathrm{~mL} / \mathrm{kg} / \mathrm{hr}$ compared with a dose $>35 \mathrm{~mL} / \mathrm{kg} / \mathrm{hr}$ after adjustment (HR, 3.13; 95\%

\section{Table 2. Causes of death for non-survivors}

\begin{tabular}{lc}
\hline Cause of death & Non-survivor $(\mathrm{n}=152)$ \\
\hline HRS or AKI & $17(11.2)$ \\
Gastrointestinal bleeding & $29(19.1)$ \\
Other LC complications $^{\mathrm{a}}$ & $55(36.2)$ \\
Infection $^{\text {Cardiovascular event }}$ & $34(22.4)$ \\
Others $^{\mathrm{b}}$ & $6(3.9)$ \\
\hline
\end{tabular}

Data are expressed as number (\%).

AKI, acute kidney injury; HRS, hepatorenal syndrome; LC, liver cirrhosis. ancludes lactic acidosis, spontaneous bacterial peritonitis, hepatic encephalopathy, hepatocellular carcinoma, and a bleeding episode due to coagulopathy except gastrointestinal bleeding. 'Includes trauma, anaphylactic shock, and unknown causes. 


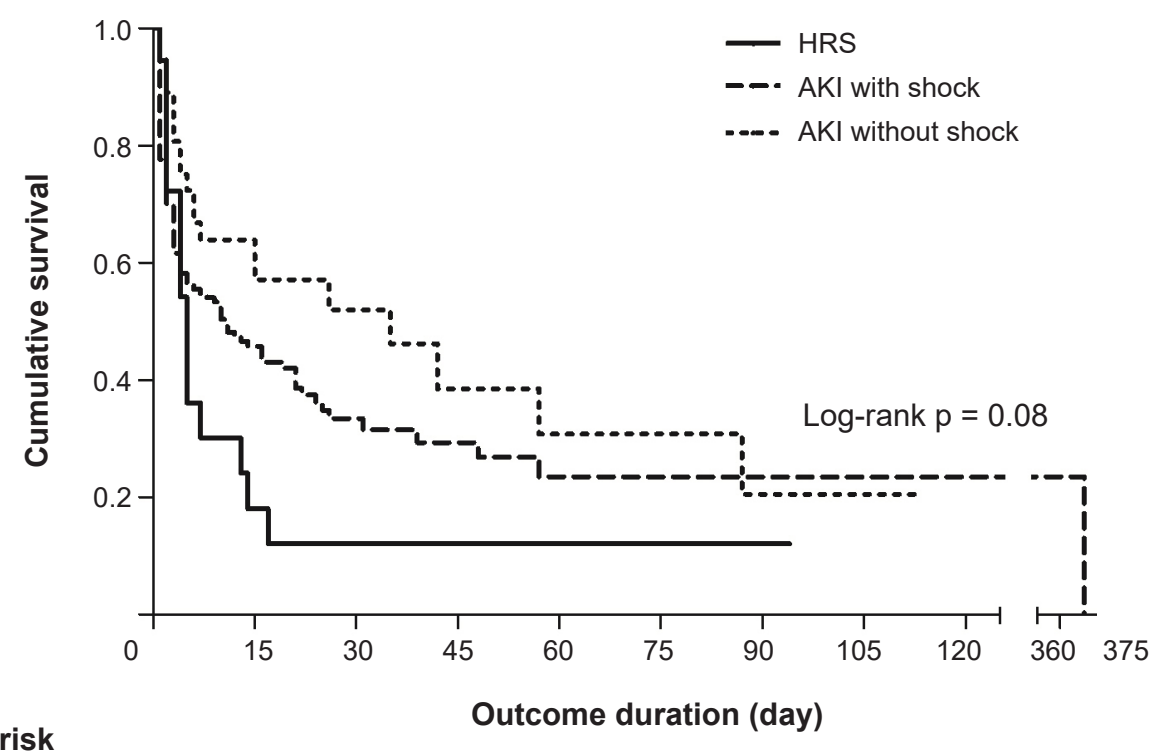

Number at risk

$\begin{array}{llllllllllll}\text { HRS } & 18 & 3 & 1 & 1 & 1 & 1 & 1 & 0 & 0 & 0 & 0 \\ \text { AKI with shock } & 172 & 53 & 19 & 13 & 7 & 6 & 4 & 3 & 1 & 1 & 0 \\ \text { AKI without shock } & 39 & 19 & 10 & 5 & 4 & 4 & 2 & 2 & 0 & 0 & 0\end{array}$

Figure 1. Kaplan-Meier curves for mortality according to causes of CRRT. Causes of CRRT were not significantly associated with inhospital mortality in liver cirrhosis patients $(p=0.08)$.

AKI, acute kidney injury; CRRT, continuous renal replacement therapy; HRS, hepatorenal syndrome.

\section{CI, 1.62-6.05; $\mathrm{p}=0.001$ ) (Table 4).}

Effects of continuous renal replacement therapy delivered dose on mortality according to liver cirrhosis severity

We divided study patients into two groups based on a MELD score of 30, which was previously reported to have a mortality rate of above $50 \%[20,21]$. ROC analysis showed that the area under the curve was $0.707(\mathrm{p}<0.001)$ and the maximum Youden's index was 28.5, which is similar to the conventional cutoff value for predicting mortality. A CRRT delivered dose $<25 \mathrm{~mL} / \mathrm{kg} / \mathrm{hr}$ was a significant risk factor for in-hospital mortality in the severe LC group after adjustment (MELD score $\geq 30$; HR, 2.80; 95\% CI, 1.32-5.96; $\mathrm{p}=0.007$ ) (Table 4). However, there was no significant correlation between CRRT delivered dose and in-hospital mortality in patients with a MELD score $<30$.

\section{Discussion}

AKI in LC patients, especially those who are not candidates for liver transplantation, is extremely challenging to treat. Several previous studies demonstrated that development of AKI was an independent prognostic factor for mortality in LC patients [12,22-25]. In the current study, we investigated the clinical characteristics of LC patients initiating CRRT and explored factors associated with in-hospital mortality. We observed that non-listed LC patients needing CRRT had an in-hospital mortality rate of $66.4 \%$, which is higher than that reported in a previous study of middle-aged (55-64 years) patients from our hospital for whom CRRT was initiated [7]. A high APACHE II score, high MELD score, and low CRRT delivered dose were significant risk factors for in-hospital mortality. The mortality rate was significantly higher in patients with a CRRT delivered dose $<25 \mathrm{~mL} / \mathrm{kg} / \mathrm{hr}$ than those with a delivered dose $>35 \mathrm{~mL} / \mathrm{kg} / \mathrm{hr}$ after adjustment. This association was significant only in patients with severe LC (MELD score $\geq 30$ ).

Previous multicenter clinical trials have investigated the relationship between CRRT dose and mortality [26,27]. These trials reported a lower mortality rate when the CRRT delivered dose was $>19-22 \mathrm{~mL} / \mathrm{kg} / \mathrm{hr}$, although the higher 
Table 3. Cox regression analysis results for in-hospital mortality

\begin{tabular}{|c|c|c|c|c|}
\hline \multirow{2}{*}{ Variable } & \multicolumn{2}{|c|}{ Univariable analysis } & \multicolumn{2}{|c|}{ Multivariable analysis } \\
\hline & $\mathrm{HR}(95 \% \mathrm{Cl})$ & p-value & $\mathrm{HR}(95 \% \mathrm{Cl})$ & p-value \\
\hline Age (per year) & $1.00(0.98-1.01)$ & 0.79 & $1.01(0.99-1.03)$ & 0.41 \\
\hline Male sex (vs. female) & $1.00(0.65-1.39)$ & 0.79 & $0.73(0.48-1.12)$ & 0.15 \\
\hline \multicolumn{5}{|l|}{ Comorbidity } \\
\hline Diabetes mellitus & $1.14(0.81-1.59)$ & 0.46 & & \\
\hline Hypertension & $0.95(0.67-1.34)$ & 0.76 & & \\
\hline Chronic kidney disease & $1.17(0.71-1.94)$ & 0.55 & & \\
\hline \multicolumn{5}{|l|}{ ICU risk factors } \\
\hline Ventilator use & $1.42(1.03-1.96)$ & 0.04 & & \\
\hline Vasopressor use & $2.47(1.67-3.67)$ & $<0.001$ & & \\
\hline SOFA score & $1.06(1.03-1.10)$ & $<0.001$ & & \\
\hline APACHE II score & $1.04(1.02-1.06)$ & 0.001 & $1.03(1.01-1.06)$ & 0.02 \\
\hline MELD score & $1.08(1.05-1.11)$ & $<0.001$ & $1.08(1.04-1.11)$ & $<0.001$ \\
\hline $\log \cup O$ & $0.90(0.83-0.98)$ & 0.011 & $0.94(0.85-1.04)$ & 0.26 \\
\hline Log CRRT duration & $0.77(0.68-0.87)$ & $<0.001$ & $0.88(0.77-1.02)$ & 0.08 \\
\hline Prescribed dose (per mL/kg/hr) & $1.00(0.94-1.01)$ & 0.23 & & \\
\hline Delivered dose (per mL/kg/hr) & $0.95(0.92-0.98)$ & 0.001 & $0.95(0.92-0.98)$ & 0.002 \\
\hline \multicolumn{5}{|l|}{ Laboratory finding } \\
\hline White blood cell (per $\left.10^{3} / \mu \mathrm{L}\right)$ & $1.01(0.99-1.03)$ & 0.22 & & \\
\hline Hemoglobin (per g/dL) & $0.98(0.91-1.05)$ & 0.55 & & \\
\hline Platelet (per $10^{3} / \mu \mathrm{L}$ ) & $1.00(1.00-1.00)$ & 0.2 & & \\
\hline Total bilirubin (per mg/dL) & $1.01(0.99-1.04)$ & 0.21 & & \\
\hline Total protein (per $\mathrm{g} / \mathrm{dL}$ ) & $0.80(0.70-0.99)$ & 0.002 & $0.95(0.80-1.12)$ & 0.55 \\
\hline Albumin (per $g / d L)$ & $0.96(0.84-1.10)$ & 0.56 & & \\
\hline Creatinine (per mg/dL) & $1.00(0.96-1.03)$ & 0.84 & & \\
\hline BUN (per mg/dL) & $1.00(0.99-1.00)$ & 0.57 & & \\
\hline Sodium (per mmol/L) & $1.04(1.01-1.06)$ & 0.001 & & \\
\hline PT-INR & $1.21(1.10-1.33)$ & $<0.001$ & & \\
\hline Total $\mathrm{CO}_{2}($ per $\mathrm{mmol} / \mathrm{L})$ & $0.99(0.97-1.02)$ & 0.46 & & \\
\hline $\mathrm{pH}$ & $0.21(0.07-0.65)$ & 0.007 & & \\
\hline
\end{tabular}

Adjusted factors are age, sex, APACHE II score, MELD score, log UO, log CRRT duration, delivered dose, and total protein.

APACHE II, Acute Physiology and Chronic Health Evaluation II; BUN, blood urea nitrogen; Cl, confidence interval; CRRT, continuous renal replacement therapy; HR, hazard ratio; ICU, intensive care unit; MELD, Model for End-Stage Liver Disease; PT-INR, prothrombin time-international normalized ratio; SOFA, Sequential Organ Failure Assessment; UO, urine output.

CRRT delivered dose group did not have decreased mortality compared to the lower CRRT delivered dose group [28]. In other words, maintaining the CRRT delivered dose in an appropriate range reduced mortality. Our results support the hypothesis that there is a minimum required CRRT delivered dose for improvement of mortality in susceptible patients.

We found that those patients with a higher MELD score group were more affected by CRRT delivered dose than those with a lower MELD score. CRRT is widely used as an extracorporeal technique to support both kidney and liver function in liver failure patients. We would expect to ob- serve an improvement in mortality rate due to a reduction in complications from accumulated toxins caused by liver dysfunction. Consistent with this, several clinical studies have reported that CRRT can reduce serum bilirubin and ammonia levels $[29,30]$. However, literature regarding the relationship between bilirubin, ammonia excretion, and mortality is sparse. Cardoso et al. [5] reported a $38 \%$ reduction in serum ammonia from day 1 to 3 as well as a reduction in 21day all-cause mortality with CRRT. In our study, because serum ammonia levels were not collected, we were unable to analyze the association between serum ammonia levels and 


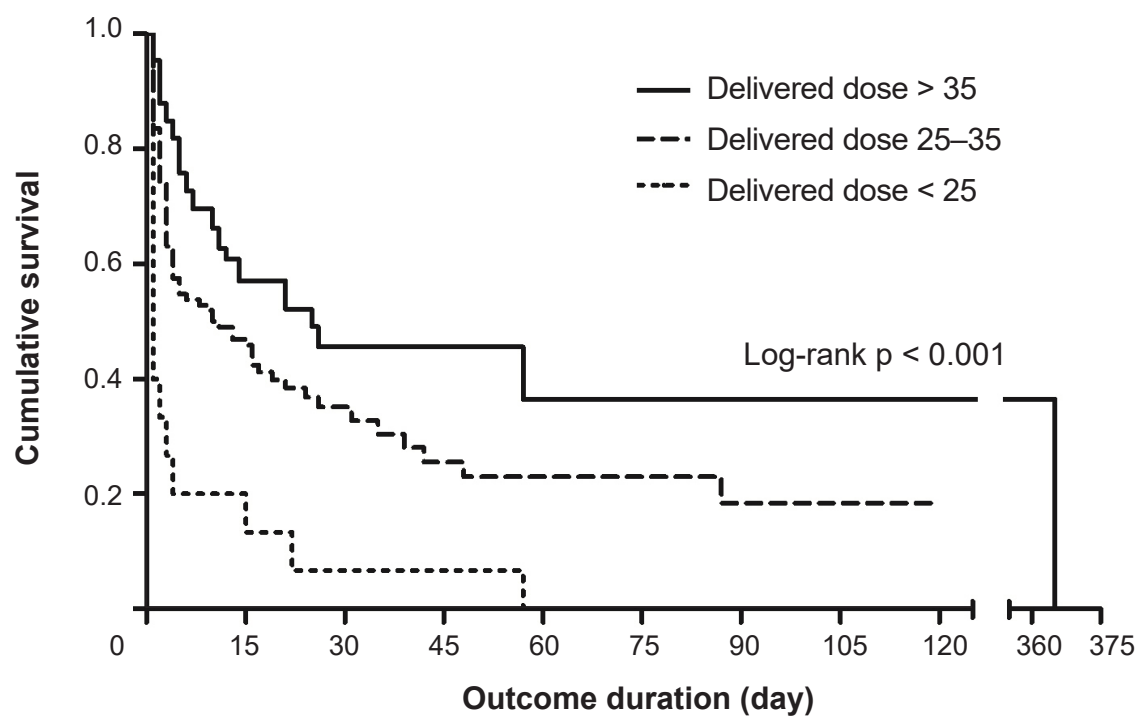

Number at risk

$\begin{array}{llllllllllll}\text { Delivered dose }>35 & 75 & 29 & 13 & 8 & 5 & 4 & 3 & 2 & 1 & 1 & 0 \\ \text { Delivered dose 25-35 } & 122 & 42 & 16 & 10 & 8 & 7 & 4 & 3 & 0 & 0 & 0 \\ \text { Delivered dose }<25 & 15 & 3 & 1 & 1 & 0 & 0 & 0 & 0 & 0 & 0 & 0\end{array}$

Figure 2. Kaplan-Meier curves for mortality stratified by CRRT delivered dose. Mortality rate differed significantly among the stratified groups according to CRRT delivered dose $(\mathrm{mL} / \mathrm{kg} / \mathrm{hr} ; \mathrm{p}<0.001)$.

CRRT, continuous renal replacement therapy.

Table 4. Effects of CRRT delivered dose on mortality according to MELD group

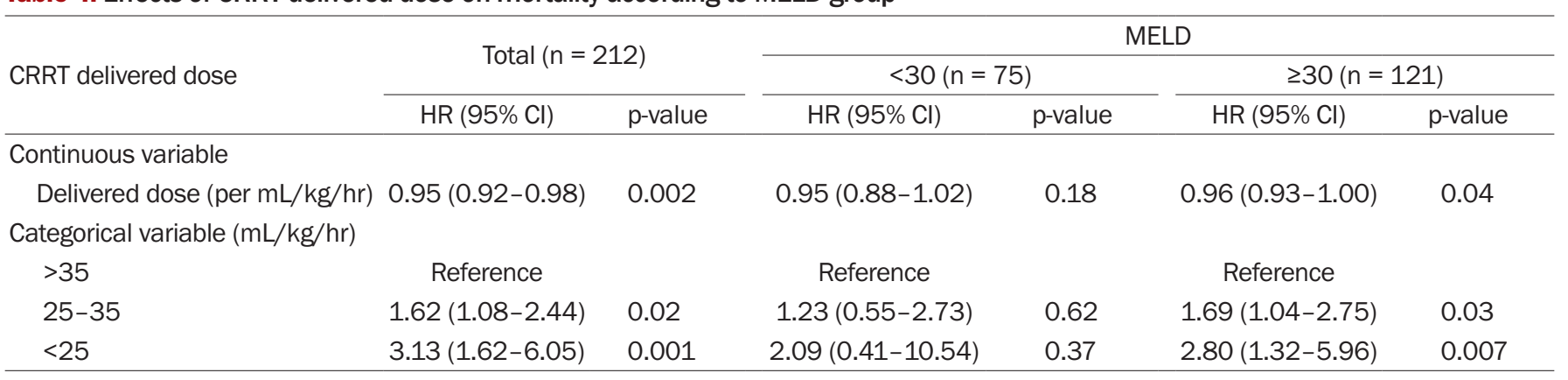

Adjusted factors are age, sex, APACHE II score, MELD score, log urine outcome, log CRRT duration, delivered dose, and total protein. $\mathrm{Cl}$, confidence interval; CRRT, continuous renal replacement therapy; HR, hazard ratio; MELD, Model for End-Stage Liver Disease.

mortality rates. Further investigation is needed to provide a rationale for advocating for CRRT for liver support in populations with high morbidity.

In CRRT treatment, an optimal prescription is important, including the appropriate CRRT dose [28]. The CRRT delivered dose is usually lower than the prescribed dose [31]. Furthermore, CRRT is often discontinued due to clotted filters or hypotension, which frequently occur in LC patients with cardiovascular instability and coagulopathy [32]. Non-sur- vivors had lower blood pressures and required more vasopressor use than survivors in our study. This may have contributed to delivery of a lower CRRT dose in non-survivors than survivors. However, there was no significant difference in CRRT downtime between the two groups, so other factors need to be considered. Prior studies have demonstrated that the discrepancy between the prescribed and delivered CRRT dose is due to multiple factors [33-35]. Malfunction of the catheter and filter may contribute to reduced blood flow and 
lead to machine disconnection from the patient. In addition, a decrease in membrane permeability, an increase in urea production, and a larger distribution volume of uremic solutes can also increase the gap between the prescribed and delivered amount of CRRT.

The non-survivor group had lower serum albumin levels and a more prolonged PT-INR than the survivor group, implying worsened LC severity in the former. Furthermore, a higher APACHE II score and higher MELD score were independent risk factors for in-hospital mortality. Our results suggest that multiorgan failure is correlated with mortality, consistent with previous studies [36,37]. In a meta-analysis, the ICU score, which refers to the degree of organ damage, was a predictive factor for short-term mortality in LC patients. In particular, liver and kidney dysfunction affect longterm mortality [38].

The survivor group had lower serum sodium levels despite a lower MELD score than the non-survivor group. There was no significant difference in serum sodium levels at hospitalization between the survivor and non-survivor groups ( $131.4 \pm 6.0$ vs. $132.9 \pm 6.6$, respectively; $\mathrm{p}=0.08$ ). This indicates that the non-survivor group had greater serum sodium fluctuations than the survivor group. In addition, a previous study showed that serum sodium variation $(\geq 6 \mathrm{mEq}$ / L) during hospital stay was a predictor of the mortality rate of hospitalized patients [39]. Thus, we further analyzed the relationship between mortality and serum sodium variation ( $\triangle \mathrm{Na}$, defined as the absolute difference between the serum sodium value at hospitalization and before CRRT initiation). Compared to the survivor group, the non-survivor group had a higher $\Delta \mathrm{Na}(2.6 \mathrm{mEq} / \mathrm{L}[0.0-7.1 \mathrm{mEq} / \mathrm{L}]$ vs. $0.9 \mathrm{mEq} /$ $\mathrm{L}[0.0-2.6 \mathrm{mEq} / \mathrm{L}], \mathrm{p}=0.002)$ and a higher proportion of patients with $\Delta \mathrm{Na} \geq 6 \mathrm{mEq} / \mathrm{L}$ (28.3\% vs. $11.7 \%$ ). In univariable analysis, $\log \Delta \mathrm{Na}$ (HR, 1.29; 95\% CI, 1.10-1.52; $\mathrm{p}=0.002)$ and $\Delta \mathrm{Na} \geq 6 \mathrm{mEq} / \mathrm{L}$ (HR, 1.73; 95\% CI, 1.21-2.47; $\mathrm{p}=0.002$ ) were significantly correlated with increased in-hospital mortality. However, multivariable analysis showed that $\log \Delta \mathrm{Na}$ (HR, 1.13; 95\% CI, 0.83-1.52; $\mathrm{p}=0.44$ ) and $\Delta \mathrm{Na} \geq 6 \mathrm{mEq} / \mathrm{L}$ (HR, 1.43; 95\% CI, 0.72-2.84; $\mathrm{p}=0.30$ ) were not significant predictors of in-hospital mortality in the present study.

Although the importance of AKI is increasingly being recognized, there are no gold-standard diagnostic criteria for AKI in LC patients. The International Club of Ascites announced new criteria for staging AKI in this population in 2015 [19]. UO has been deleted from the 2015 ICA-AKI diagnostic criteria because cirrhotic patients are frequently oliguric with sodium retention, even though kidney function is normal [19]. Although UO for 6 hours before CRRT was lower in non-survivors than in survivors, this was not correlated with mortality rate. However, measurement of UO for only six hours immediately before CRRT may be too short a time window. In addition, UO does not fully reflect deterioration of kidney function in LC patients, so CRRT should be initiated based on consideration of the dysfunction of various organs. Consequently, more meticulous management of LC patients with severe acute illness is required. Clinicians should modify the precipitating factors of AKI, restore intravascular volume with albumin infusion with continuous assessment of organ dysfunction, and assess the need for CRRT.

Reasons for initiating CRRT were not significantly correlated with mortality, consistent with a previous study. Allegretti et al. [14] reported that the 6-month mortality rate in KRT was $85 \%$, and that the cause of AKI (ATN vs. HRS) was not associated with mortality in non-listed LC patients requiring KRT. However, this finding should be interpreted with caution. Most existing studies have been retrospective in nature, involving patients with different baseline characteristics, CRRT initiation criteria, and prescriptions. In addition, there may have been some difficulties in clearly distinguishing categories of HRS and AKI, although there are HRS diagnostic criteria for LC patients [19].

In a previous meta-analysis of LC patients admitted to the ICU, patients admitted for variceal bleeding had a lower mortality rate than patients admitted for other reasons [38]. In our study, there were no significant associations between the cause of admission (i.e., gastrointestinal bleeding, HRS or AKI, other LC complications, infection, cardiovascular events) and in-hospital mortality ( $p=0.22)$. Our study findings may be different from those of previous studies because we focused on critically ill patients who needed CRRT.

We conducted this study because previous studies on CRRT outcomes of non-listed LC patients have reported inconsistent findings, and Korean data are insufficient. However, there are several limitations to our study. First, as a retrospective observational study, potential biases that we did not account for may have been present. Second, we collected data from two tertiary hospitals with liver transplantation centers, but not within the same time period. Finally, there might have been variability in clinical diagnoses, timing of CRRT, 
and prescription for CRRT among the treating physicians.

In conclusion, the outcomes of LC patients needing CRRT remain poor. High APACHE II score, high MELD score, and low CRRT delivered dose were significant risk factors for increased in-hospital mortality in LC patients requiring CRRT. A low CRRT delivered dose had a greater impact on in-hospital mortality in patients with high LC severity than those with low LC severity. Therefore, achieving an effective CRRT dose is crucial, especially in patients with severe LC.

\section{Conflicts of interest}

All authors have no conflicts of interest to declare.

\section{Funding}

This work was supported by clinical research grant from $\mathrm{Pu}$ san National University Hospital in 2020.

\section{Authors' contributions}

Conceptualization: YHJ, IYK, SHS, HJK

Data curation, Formal analysis: YHJ, IYK, GSJ, SHS, HJK

Funding acquisition: HJK

Investigation: All authors

Project administration: HJK

Writing-original draft: YHJ, IYK, HJK

Writing-review \& editing: All authors

All authors read and approved the final manuscript.

\section{ORCID}

You Hyun Jeon, https://orcid.org/0000-0001-7318-5753 Il Young Kim, https://orcid.org/0000-0002-1731-6357 Gum Sook Jang, https://orcid.org/0000-0003-2061-9305 Sang Heon Song, https://orcid.org/0000-0002-8218-6974 Eun Young Seong, https://orcid.org/0000-0002-6006-0051 Dong Won Lee, https://orcid.org/0000-0003-0282-484X Soo Bong Lee, https://orcid.org/0000-0002-3388-7993 Hyo Jin Kim, https://orcid.org/0000-0001-9289-9073

\section{References}

1. Wong F. Acute kidney injury in liver cirrhosis: new definition and application. Clin Mol Hepatol 2016;22:415-422.
2. Wald R, McArthur E, Adhikari NK, et al. Changing incidence and outcomes following dialysis-requiring acute kidney injury among critically ill adults: a population-based cohort study. Am J Kidney Dis 2015;65:870-877.

3. Davenport A. AKI in a patient with cirrhosis and ascites. Clin J Am Soc Nephrol 2012;7:2041-2048.

4. Wright G, Noiret L, Olde Damink SW, Jalan R. Interorgan ammonia metabolism in liver failure: the basis of current and future therapies. Liver Int 2011;31:163-175.

5. Cardoso FS, Gottfried M, Tujios S, Olson JC, Karvellas CJ; US Acute Liver Failure Study Group. Continuous renal replacement therapy is associated with reduced serum ammonia levels and mortality in acute liver failure. Hepatology 2018;67:711-720.

6. Park S, Lee S, Jo HA, et al. Epidemiology of continuous renal replacement therapy in Korea: results from the National Health Insurance Service claims database from 2005 to 2016. Kidney Res Clin Pract 2018;37:119-129.

7. Rhee H, Jang KS, Park JM, et al. Short- and long-term mortality rates of elderly acute kidney injury patients who underwent continuous renal replacement therapy. PLoS One 2016;11:e0167067.

8. Fraley DS, Burr R, Bernardini J, Angus D, Kramer DJ, Johnson JP. Impact of acute renal failure on mortality in end-stage liver disease with or without transplantation. Kidney Int 1998;54:518524.

9. Witzke O, Baumann M, Patschan D, et al. Which patients benefit from hemodialysis therapy in hepatorenal syndrome? J Gastroenterol Hepatol 2004;19:1369-1373.

10. Thorat A, Jeng LB. Management of renal dysfunction in patients with liver cirrhosis: role of pretransplantation hemodialysis and outcomes after liver transplantation. Semin Vasc Surg 2016;29:227-235.

11. Belcher JM. Is there a role for dialysis in patients with hepatorenal syndrome who are not liver transplant candidates? Semin Dial 2014;27:288-291.

12. Umemura T, Joshita S, Shibata S, et al. Renal impairment is associated with increased risk of mortality in patients with cirrhosis: a retrospective cohort study. Medicine (Baltimore) 2019;98:e14475.

13. Shetty S, Nagaraju SP, Shenoy S, et al. Acute kidney injury in patients with cirrhosis of liver: clinical profile and predictors of outcome. Indian J Gastroenterol 2018;37:248-254.

14. Allegretti AS, Parada XV, Eneanya ND, et al. Prognosis of patients with cirrhosis and AKI who initiate RRT. Clin J Am Soc Nephrol 2018;13:16-25.

15. Xiong J, Pu L, Xiong H, et al. Evaluation of the criteria of hepa- 
torenal syndrome type of acute kidney injury in patients with cirrhosis admitted to ICU. Scand J Gastroenterol 2018;53:15901596.

16. Cárdenas-Turanzas M, Ensor J, Wakefield C, et al. Cross-validation of a Sequential Organ Failure Assessment score-based model to predict mortality in patients with cancer admitted to the intensive care unit. J Crit Care 2012;27:673-680.

17. Capuzzo M, Valpondi V, Sgarbi A, et al. Validation of severity scoring systems SAPS II and APACHE II in a single-center population. Intensive Care Med 2000;26:1779-1785.

18. Kim WR, Biggins SW, Kremers WK, et al. Hyponatremia and mortality among patients on the liver-transplant waiting list. $N$ Engl J Med 2008;359:1018-1026.

19. Angeli $\mathrm{P}$, Ginès $\mathrm{P}$, Wong $\mathrm{F}$, et al. Diagnosis and management of acute kidney injury in patients with cirrhosis: revised consensus recommendations of the International Club of Ascites. J Hepatol 2015;62:968-974.

20. Kamath PS, Kim WR; Advanced Liver Disease Study Group. The model for end-stage liver disease (MELD). Hepatology 2007;45:797-805.

21. Aiello FI, Bajo M, Marti F, Gadano A, Musso CG. Model for Endstage Liver Disease (MELD) score and liver transplant: benefits and concerns. AME Med J 2017;2:168.

22. Nuthalapati A, Schluterman N, Khanna A, Greenberg D, Thuluvath PJ. Impact of acute kidney injury on mortality of patients hospitalized for complications of cirrhosis. J Clin Exp Hepatol 2017;7:290-299.

23. Tandon P, James MT, Abraldes JG, Karvellas CJ, Ye F, Pannu N. Relevance of new definitions to incidence and prognosis of acute kidney injury in hospitalized patients with cirrhosis: a retrospective population-based cohort study. PLoS One 2016;11:e0160394.

24. Fede G, D'Amico G, Arvaniti V, et al. Renal failure and cirrhosis: a systematic review of mortality and prognosis. $J$ Hepatol 2012;56:810-818.

25. Gessolo Lins PR, Carvalho Padilha WS, Magalhaes Giradin Pimentel CF, Costa Batista M, Teixeira de Gois AF. Risk factors, mortality and acute kidney injury outcomes in cirrhotic patients in the emergency department. BMC Nephrol 2018;19:277.

26. VA/NIH Acute Renal Failure Trial Network; Palevsky PM, Zhang $\mathrm{JH}$, et al. Intensity of renal support in critically ill patients with acute kidney injury. N Engl J Med 2008;359:7-20.
27. RENAL Replacement Therapy Study Investigators; Bellomo R, Cass A, et al. Intensity of continuous renal-replacement therapy in critically ill patients. N Engl J Med 2009;361:1627-1638.

28. Kellum JA, Ronco C. Dialysis: results of RENAL: what is the optimal CRRT target dose? Nat Rev Nephrol 2010;6:191-192.

29. Nand N, Verma P, Jain D. Comparative evaluation of continuous veno-venous hemodiafiltration and continuous arterio-venous hemodiafiltration in patients of hepatic failure and / or hepatorenal syndrome. J Assoc Physicians India 2019;67:39-42.

30. Naorungroj T, Yanase F, Eastwood GM, Baldwin I, Bellomo R. Extracorporeal ammonia clearance for hyperammonemia in critically ill patients: a scoping review. Blood Purif 2020;1-9.

31. Macedo E, Claure-Del Granado R, Mehta RL. Effluent volume and dialysis dose in CRRT: time for reappraisal. Nat Rev Nephrol 2011;8:57-60.

32. Agarwal B, Shaw S, Shankar Hari M, Burroughs AK, Davenport A. Continuous renal replacement therapy (CRRT) in patients with liver disease: is circuit life different? J Hepatol 2009;51:504-509.

33. Claure-Del Granado R. Dose in continuous renal replacement therapy. Gac Med Mex 2018;154(Supp 1):S40-S47.

34. Claure-Del Granado R, Macedo E, Chertow GM, et al. Effluent volume in continuous renal replacement therapy overestimates the delivered dose of dialysis. Clin J Am Soc Nephrol 2011;6:467475.

35. Marshall MR. Current status of dosing and quantification of acute renal replacement therapy. Part 1: mechanisms and consequences of therapy under-delivery. Nephrology (Carlton) 2006;11:171-180.

36. Levesque E, Saliba F, Ichaï P, Samuel D. Outcome of patients with cirrhosis requiring mechanical ventilation in ICU.J Hepatol 2014;60:570-578.

37. Annamalai A, Harada MY, Chen M, et al. Predictors of mortality in the critically ill cirrhotic patient: is the model for end-stage liver disease enough? J Am Coll Surg 2017;224:276-282.

38. Weil D, Levesque E, McPhail M, et al. Prognosis of cirrhotic patients admitted to intensive care unit: a meta-analysis. Ann Intensive Care 2017;7:33.

39. Thongprayoon C, Cheungpasitporn W, Yap JQ, Qian Q. Increased mortality risk associated with serum sodium variations and borderline hypo- and hypernatremia in hospitalized adults. Nephrol Dial Transplant 2020;35:1746-1752. 